\title{
Base-Mediated Aerobic Oxidation of Hagemann's Ester: Competitive Hydroxylation at C-1 and C-3 Positions
}

\author{
Sung Hwan Kim, Hyun Seung Lee, Bo Ram Park, and Jae Nyoung Kim* \\ Department of Chemistry and Institute of Basic Science, Chonnam National University, Gwangju 500-757, Korea \\ *E-mail:kimjn@chonnam.ac.kr \\ Received February 13, 2011, Accepted March 2, 2011
}

Key Words : Aerobic oxidation, Hagemann's ester, Hydroxylation, Catechol

The Hagemann's ester (Fig. 1) has been extensively used for the synthesis of various complex compounds. ${ }^{1,2}$ The reason might be due to its highly-functionalized nature and ready availability. ${ }^{1,2}$ The reaction of Hagemann's ester and an electrophile can occur theoretically at C-1, C-3, C-5, and C-2' positions (Fig. 1). As an example, however, it has been generally accepted that the site preference for alkylation was $\mathrm{C}-3$, the resulting C-3-alkyl derivatives often being accompanied by small amounts of the C-1-alkyl products. ${ }^{\text {1a }}$ Although the Hagemann's ester could be air-oxidized to poly-substituted phenol or catechol derivatives, a small piece of work has been reported, ${ }^{3}$ to the best of our knowledge. Irie and co-workers examined an aerobic oxidation under the influence of $\mathrm{KF} / \mathrm{DMSO} / \mathrm{O}_{2}$ at $60{ }^{\circ} \mathrm{C}$ to obtain $\mathrm{C}-1$ oxidation product $(62 \%)$ along with a trace amount of $\mathrm{C}-3$ oxidation product $(1 \%) .{ }^{3 a}$ Stoodley and co-workers obtained a C-1 oxidation product only under the influence of excess amounts of activated charcoal ( 3 days, reflux, 50\%). ${ }^{3 b}$

Recently, we have synthesized many interesting compounds using an aerobic oxidation under the influence of a base such as DBU and $\mathrm{K}_{2} \mathrm{CO}_{3} .{ }^{4}$ Meantime we decided to examine the aerobic oxidation of Hagemann's ester under basic conditions. We imagined that $\mathrm{C}-1$ oxidation would produce either 3a or $4 \mathbf{a}$, while $\mathbf{2 a}$ could be produced by C-3 oxidation, as shown in Scheme 1. Thus we prepared compound 1a from benzaldehyde and methyl acetoacetate according to the reported procedure as a cis/trans mixture (ca. 1:8), ${ }^{1 \mathrm{~b}, 1 \mathrm{e}, 2 \mathrm{c}, 2 \mathrm{~h}}$ and examined an aerobic oxidation. Initial experiment of $\mathbf{1 a}$ with $\mathrm{K}_{2} \mathrm{CO}_{3} / \mathrm{DMF}$ under $\mathrm{O}_{2}$ balloon atmosphere produced 2a $(25 \%)$ and 3a (35\%) in moderate yields. We could not obtain a phenol derivative $\mathbf{4 a}$ at all. Aerobic oxidation of $\mathbf{1 a}$, most likely via the carbanion intermediate I, would produce II and 3a. The intermediate II was converted to a catechol derivative $\mathbf{2 a}$ by tautomerization and concomitant aerobic oxidation.

The ratio of $\mathbf{2 a} / \mathbf{3 a}$ as well as the combined yields could be improved by modifying the reaction conditions, thus we examined various aerobic oxidation conditions, as shown in Table 1. However, the use of $\mathrm{K}_{2} \mathrm{CO}_{3}, \mathrm{DBU}$, and TBAF (entries 1-3) afforded similar yields of products (60-67\%), while the use of imidazole and pyridine was not effective (entries 4 and 5). Solvent effect was also negligible (entries 6-8), unfortunately. Although the difference is small we chose the conditions in entry 7 as the optimum one based on the combined yields.

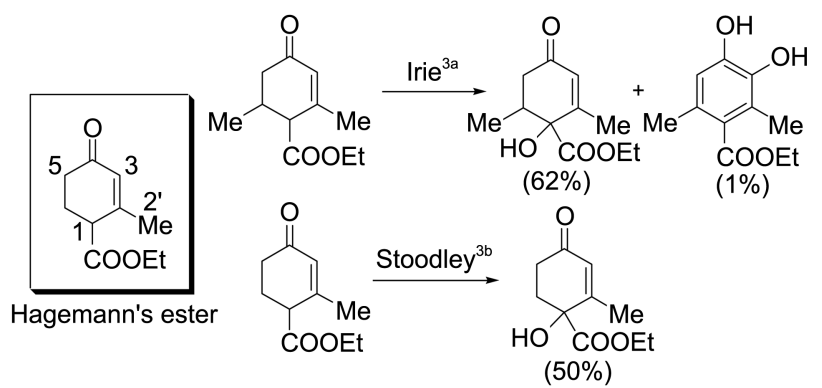

Figure 1. Typical Hagemann's ester and precedent oxidation results.

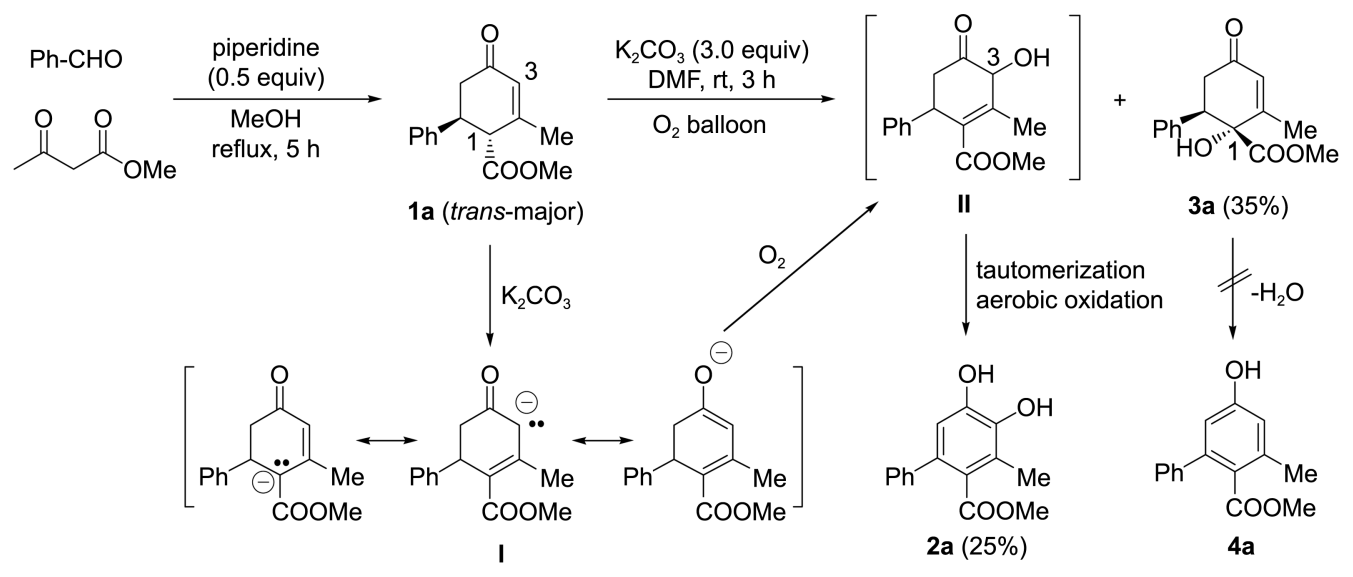

Scheme 1 
Table 1. Oxidation of 1a under various conditions

\begin{tabular}{clc}
\hline Entry & Conditions $^{a}$ & $\mathbf{2 a}(\%)^{b} / \mathbf{3 a}(\%)^{b}$ \\
\hline 1 & $\mathrm{~K}_{2} \mathrm{CO}_{3}$ (3.0 equiv), DMF, rt, 3 h & $25 / 35$ \\
2 & $\mathrm{DBU}$ (3.0 equiv), DMF, rt, 3 h & $29 / 38$ \\
3 & TBAF (3.0 equiv), THF, rt, 3 h & $24 / 40$ \\
4 & Imidazole (3.0 equiv), DMF, rt, 24 h & trace / trace \\
5 & pyridine, $100{ }^{\circ} \mathrm{C} 12 \mathrm{~h}$ & $0 / 0$ \\
6 & DBU (3.0 equiv), $\mathrm{CH}_{3} \mathrm{CN}, \mathrm{rt}, 3 \mathrm{~h}$ & $20 / 31$ \\
7 & DBU (2.0 equiv), $\mathrm{CH}_{2} \mathrm{Cl}_{2}, \mathrm{rt}, 5 \mathrm{~h}$ & $27 / 48$ \\
8 & DBU (0.5 equiv), DMF, rt, 5 h & $23 / 40$ \\
\hline
\end{tabular}

${ }^{a}$ Carried out under $\mathrm{O}_{2}$ balloon. ${ }^{b}$ Isolated yield.

In order to check the generality, starting materials $\mathbf{1 b}$-h were prepared $^{1,2}$ and examined an aerobic oxidation under the optimum conditions. The results are summarized in Table 2 . In the reactions of 6-aryl derivatives (entries 1-5), the yields of catechol derivatives 2a-e were low (19-27\%), while the yields of $\mathrm{C}-1$ oxidation products 3a-e were moderate $(45-52 \%)$. The reaction of 6-pentyl derivative $\mathbf{1 f}$ produced 3f (59\%) as a major product (entry 6). Trace amount of $2 \mathbf{f}$ was observed on TLC; however, we could not obtain $2 \mathbf{f}$ in appreciable amounts. The reaction of 6-methyl derivative $\mathbf{1 g}$ also afforded $\mathbf{3 g}(61 \%)$ as a major product (entry 7), and this result is similar to that of Irie. ${ }^{3 a}$ Quite surprisingly, the reaction of $\mathbf{1 h}$ produced catechol $\mathbf{2 h}$ as a major product in moderate yield ( $47 \%$, entry 8$)$. The different

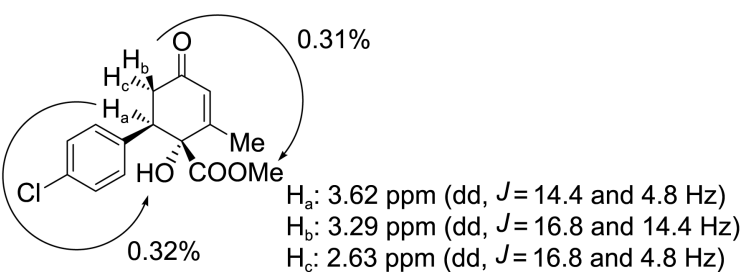

Figure 2. NOE results of compound $\mathbf{3 d}$.

reactivity of $\mathbf{1 h}$ is not clear at this stage..$^{5}$

It is interesting to note that compounds 3a-f were obtained as single diastereomers although we used cis/trans mixtures of 1a-f. ${ }^{3 a}$ The only exception was 6-methyl derivative $\mathbf{3 g}$ (entry 7). A trace amount of the other diastereomer was contaminated (ca. 10\%). The stereochemistry of 3a-f is thought to be as that shown in Figure 2, tentatively. ${ }^{6} \mathrm{NOE}$ experimental results with 3d stated that the substituent at C6 and an ester moiety are positioned in a cis-relationship.

A useful synthetic application of C-1 oxidation products was demonstrated in Scheme 2, with 3a as a representative example. ${ }^{7}$ Treatment of 3a with NaI in DMSO $\left(120{ }^{\circ} \mathrm{C}, 30\right.$ min) produced 5a via $\mathrm{NaI} / \mathrm{O}_{2}$-assisted concomitant dealkoxycarbonylation/aerobic oxidation. ${ }^{8}$

In summary, we examined an aerobic oxidation of various Hagemann's ester under the influence of $\mathrm{DBU}$ in $\mathrm{CH}_{2} \mathrm{Cl}_{2}$. Hydroxylation at $\mathrm{C}-1$ position is favored to produce 1-

Table 2. DBU-mediated aerobic oxidation of Hagemann's ester

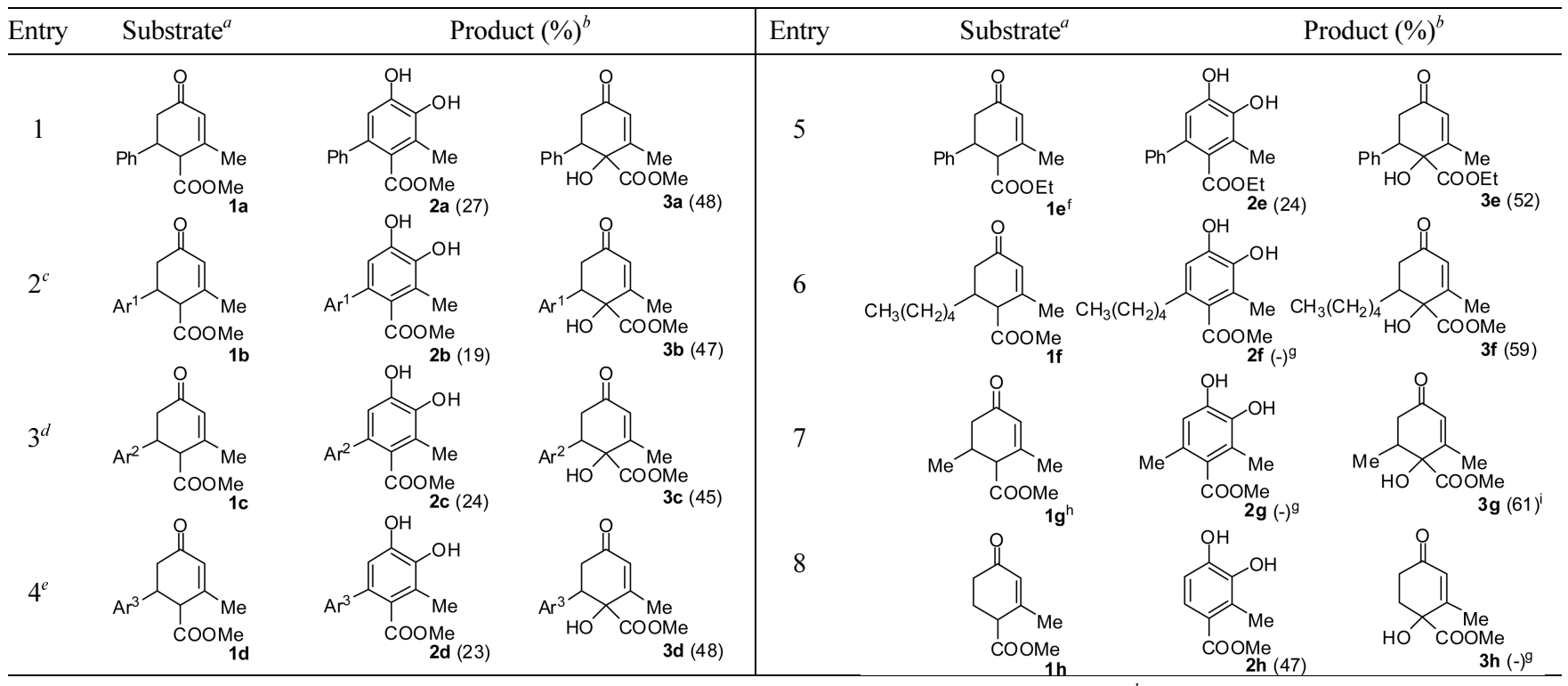

${ }^{a}$ Conditions: aldehyde $(2.0 \mathrm{mmol})$, methyl acetoacetate ( 2.5 equiv), piperidine ( 0.5 equiv), $\mathrm{MeOH}$, reflux, 5 h. ${ }^{b} \mathrm{Conditions}$ : $\mathrm{DBU}\left(2.0\right.$ equiv), $\mathrm{CH}_{2} \mathrm{Cl}_{2}, \mathrm{rt}$, $5 \mathrm{~h}, \mathrm{O}_{2}$ balloon. ${ }^{c} \mathrm{Ar}^{1}$ is $4-\mathrm{MePh} .{ }^{d} \mathrm{Ar}^{2}$ is 4-MeOPh.${ }^{e} \mathrm{Ar}{ }^{3}$ is $4-\mathrm{ClPh} .{ }^{\circ}$ Ethyl acetoacetate was used. ${ }^{g}$ Failed to isolate. ${ }^{h} \mathrm{Conditions}$ : rt, 2 days. ${ }^{i} \mathrm{Trace}$ amount (ca. 10\%) of the other stereoisomer was mixed.

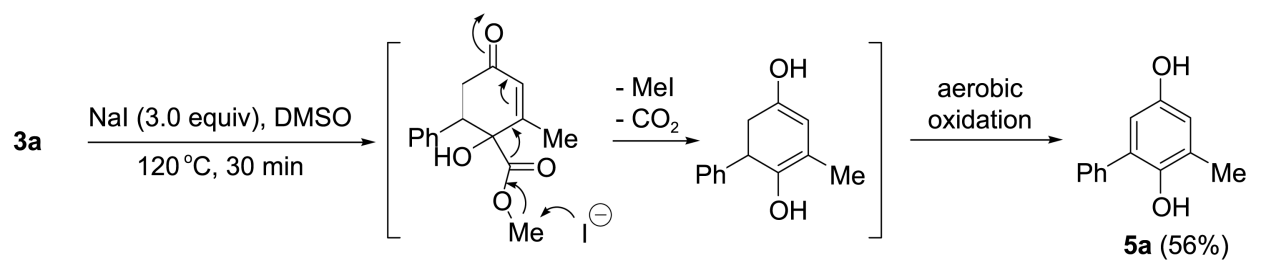

Scheme 2 
hydroxycyclohexenone derivatives in moderate yields, while hydroxylation at C-3 position occurred as a minor pathway.

\section{Experimental Section}

Typical Procedure for the Preparation of 1a. A stirred solution of benzaldehyde $(212 \mathrm{mg}, 2.0 \mathrm{mmol})$, methyl acetoacetate (580 mg, $5.0 \mathrm{mmol})$, and piperidine $(85 \mathrm{mg}, 1.0 \mathrm{mmol})$ in $\mathrm{MeOH}(6 \mathrm{~mL})$ was heated to reflux for $5 \mathrm{~h}$. After aqueous extractive workup and column chromatographic purification process (hexanes/EtOAc, 6:1) compound 1a was isolated a white solid, $342 \mathrm{mg}(70 \%){ }^{1 \mathrm{~b}, 1 \mathrm{e}, 2 \mathrm{c}, 2 \mathrm{~h}}$ Other Hagemann's esters (1b-d, $\mathbf{1 e},^{2 \mathrm{~d}-\mathrm{g}} \mathbf{1 f},{ }^{2 \mathrm{j}} \mathbf{1 g},{ }^{2 \mathrm{c}} \mathbf{1 h}^{2 \mathrm{~d}-\mathrm{g}}$ ) were prepared similarly, and the spectroscopic data of unknown compounds, $\mathbf{1 b}$-d are as follows.

Compound 1b: $60 \%$; white solid, mp $101-102{ }^{\circ} \mathrm{C}$; IR (KBr) $1737,1674,1632,1435 \mathrm{~cm}^{-1} ;{ }^{1} \mathrm{H}$ NMR $\left(\mathrm{CDCl}_{3}, 300 \mathrm{MHz}\right) \delta$ $1.96(\mathrm{~s}, 3 \mathrm{H}), 2.32(\mathrm{~s}, 3 \mathrm{H}), 2.57-2.74(\mathrm{~m}, 2 \mathrm{H}), 3.55-3.68(\mathrm{~m}$, $2 \mathrm{H}), 3.61(\mathrm{~s}, 3 \mathrm{H}), 6.05(\mathrm{~s}, 1 \mathrm{H}), 7.09(\mathrm{~d}, J=8.7 \mathrm{~Hz}, 2 \mathrm{H}), 7.12$ $(\mathrm{d}, J=8.7 \mathrm{~Hz}, 2 \mathrm{H}) ;{ }^{13} \mathrm{C} \mathrm{NMR}\left(\mathrm{CDCl}_{3}, 75 \mathrm{MHz}\right) \delta 21.00,22.42$, 42.67, 43.42, 52.15, 54.34, 126.90, 128.37, 129.43, 137.02, 137.83, 155.97, 171.85, 197.39; ESIMS $m / z 259\left(\mathrm{M}^{+}+\mathrm{H}\right)$.

Compound 1c: $61 \%$; white solid, mp $74-75^{\circ} \mathrm{C}$; IR (KBr) $1736,1672,1612,1514,1436,1254 \mathrm{~cm}^{-1} ;{ }^{1} \mathrm{H} \mathrm{NMR}\left(\mathrm{CDCl}_{3}\right.$, $300 \mathrm{MHz}) \delta 1.96(\mathrm{~s}, 3 \mathrm{H}), 2.56-2.73(\mathrm{~m}, 2 \mathrm{H}), 3.53-3.66(\mathrm{~m}$, $2 \mathrm{H}), 3.60(\mathrm{~s}, 3 \mathrm{H}), 3.79(\mathrm{~s}, 3 \mathrm{H}), 6.05(\mathrm{~s}, 1 \mathrm{H}), 6.85(\mathrm{~d}, J=8.4$ $\mathrm{Hz}, 2 \mathrm{H}), 7.13(\mathrm{~d}, J=8.4 \mathrm{~Hz}, 2 \mathrm{H}) ;{ }^{13} \mathrm{C} \mathrm{NMR}\left(\mathrm{CDCl}_{3}, 75\right.$ $\mathrm{MHz}) \delta 22.36,42.84,43.12,52.13,54.54,55.16,114.06$, $128.07,128.36,132.88,156.03,158.68,171.88,197.39$; ESIMS $m / z 275\left(\mathrm{M}^{+}+\mathrm{H}\right)$.

Compound 1d: $59 \%$; white solid, mp 106-107 ${ }^{\circ} \mathrm{C}$; IR (KBr) 1735, 1669, 1631, 1492, $1435 \mathrm{~cm}^{-1} ;{ }^{1} \mathrm{H}$ NMR $\left(\mathrm{CDCl}_{3}, 300\right.$ MHz) $\delta 1.97(\mathrm{~s}, 3 \mathrm{H}), 2.55-2.74(\mathrm{~m}, 2 \mathrm{H}), 3.54-3.70(\mathrm{~m}, 2 \mathrm{H})$, $3.62(\mathrm{~s}, 3 \mathrm{H}), 6.06(\mathrm{~s}, 1 \mathrm{H}), 7.16(\mathrm{~d}, J=8.4 \mathrm{~Hz}, 2 \mathrm{H}), 7.29(\mathrm{~d}, J=$ $8.4 \mathrm{~Hz}, 2 \mathrm{H}) ;{ }^{13} \mathrm{C} \mathrm{NMR}\left(\mathrm{CDCl}_{3}, 75 \mathrm{MHz}\right) \delta 22.35,42.38,43.17$, 52.22, 53.99, 128.37, 128.44, 128.92, 133.13, 139.31, 155.72, 171.52, 196.67; ESIMS $m / z 279\left(\mathrm{M}^{+}+\mathrm{H}\right), 281\left(\mathrm{M}^{+}+2+\mathrm{H}\right)$.

Typical Procedure for the Synthesis of $2 \mathrm{a}$ and $3 \mathrm{a}$. To a stirred solution of $1 \mathbf{a}(244 \mathrm{mg}, 1.0 \mathrm{mmol})$ in $\mathrm{CH}_{2} \mathrm{Cl}_{2}(2 \mathrm{~mL})$ was added DBU (304 mg, $2.0 \mathrm{mmol}$ ), and the reaction mixture was stirred at room temperature for $5 \mathrm{~h}$ under $\mathrm{O}_{2}$ balloon atmosphere. After aqueous extractive workup and column chromatographic purification process (hexanes/EtOAc, 4:1) compounds 2a (70 mg, 27\%) and 3a (126 mg, 48\%) were isolated. Other entries were carried out similarly, and the spectroscopic data of unknown compounds, $\mathbf{2} \mathbf{a}-\mathbf{e}, \mathbf{2 h}$ and 3a-g are as follows.

Compound 2a: 27\%; pale yellow oil; IR (film) 3397, 1697, 1611, $1489 \mathrm{~cm}^{-1}$; ${ }^{1} \mathrm{H}$ NMR $\left(\mathrm{CDCl}_{3}, 300 \mathrm{MHz}\right) \delta 2.26(\mathrm{~s}, 3 \mathrm{H})$, 3.55 (s, 3H), 5.49 (br s, 1H), 6.08 (br s, 1H), 6.68 (s, 1H), 7.25$7.36(\mathrm{~m}, 5 \mathrm{H}) ;{ }^{13} \mathrm{C} \mathrm{NMR}\left(\mathrm{CDCl}_{3}, 75 \mathrm{MHz}\right) \delta 12.76,52.06$, 114.03, 122.75, 126.07, 127.01, 128.04, 128.20, 133.09, 140.70, 141.67, 144.14, 171.04; ESIMS m/z $259\left(\mathrm{M}^{+}+\mathrm{H}\right)$. Anal. Calcd for $\mathrm{C}_{15} \mathrm{H}_{14} \mathrm{O}_{4}$ : C, 69.76; H, 5.46. Found: C, 69.99; H, 5.62.

Compound 3a: $48 \%$; white solid, mp $117-118^{\circ} \mathrm{C}$; IR (KBr) 3495, 1736, 1670, 1437, $1234 \mathrm{~cm}^{-1}$; ${ }^{1} \mathrm{H}$ NMR $\left(\mathrm{CDCl}_{3}, 300\right.$ MHz) $\delta 1.94$ (s, 3H), 2.65 (dd, $J=16.8$ and $4.5 \mathrm{~Hz}, 1 \mathrm{H}), 3.35$ (dd, $J=16.8$ and $14.7 \mathrm{~Hz}, 1 \mathrm{H}), 3.64$ (dd, $J=14.7$ and $4.5 \mathrm{~Hz}$,
$1 \mathrm{H}), 3.77(\mathrm{~s}, 3 \mathrm{H}), 3.82(\mathrm{~s}, 1 \mathrm{H}), 6.10(\mathrm{~s}, 1 \mathrm{H}), 7.18-7.21(\mathrm{~m}, 2 \mathrm{H})$, 7.29-7.33 (m, 3H); ${ }^{13} \mathrm{C} \mathrm{NMR}\left(\mathrm{CDCl}_{3}, 75 \mathrm{MHz}\right) \delta 18.86,39.79$, $50.15,53.55,78.89,128.03,128.26,128.47,129.29$, 136.92, 158.60, 172.23, 197.79; ESIMS $m / z 261\left(\mathrm{M}^{+}+\mathrm{H}\right)$. Anal. Calcd for $\mathrm{C}_{15} \mathrm{H}_{16} \mathrm{O}_{4}$ : C, 69.22; H, 6.20. Found: $\mathrm{C}, 69.51 ; \mathrm{H}, 6.07$.

Compound 2b: 19\%; white solid, mp 164-166 ${ }^{\circ} \mathrm{C}$; IR (KBr) 3131, 1719, 1604, 1501, $1299 \mathrm{~cm}^{-1} ;{ }^{1} \mathrm{H} \mathrm{NMR}\left(\mathrm{CDCl}_{3}\right.$ + DMSO-d $\left.d_{6}, 300 \mathrm{MHz}\right) \delta 2.26(\mathrm{~s}, 3 \mathrm{H}), 2.35(\mathrm{~s}, 3 \mathrm{H}), 3.56(\mathrm{~s}$, $3 \mathrm{H}), 6.60$ (br s, 1H), 6.75 (s, 1H), 7.13 (d, $J=8.4 \mathrm{~Hz}, 2 \mathrm{H})$, $7.19(\mathrm{~d}, J=8.4 \mathrm{~Hz}, 2 \mathrm{H}), 8.70(\mathrm{br} \mathrm{s}, 1 \mathrm{H}) ;{ }^{13} \mathrm{C} \mathrm{NMR}\left(\mathrm{CDCl}_{3}+\right.$ DMSO- $\left.d_{6}, 75 \mathrm{MHz}\right) \delta 12.79,20.97,51.58,114.03,122.59$, 125.49 , 127.82, 128.74, 132.23, 136.20, 138.18, 141.99, 144.76, 170.46; ESIMS $m / z 273\left(\mathrm{M}^{+}+\mathrm{H}\right)$. Anal. Calcd for $\mathrm{C}_{16} \mathrm{H}_{16} \mathrm{O}_{4}$ : C, 70.57; H, 5.92. Found: C, 70.34; H, 6.05.

Compound 3b: 47\%; white solid, mp 110-111 ${ }^{\circ} \mathrm{C}$; IR (KBr) 3367, 1736, 1666, 1434, $1235 \mathrm{~cm}^{-1}$; ${ }^{1} \mathrm{H} \mathrm{NMR}\left(\mathrm{CDCl}_{3}\right.$, $300 \mathrm{MHz}) \delta 1.93(\mathrm{~s}, 3 \mathrm{H}), 2.33(\mathrm{~s}, 3 \mathrm{H}), 2.62(\mathrm{dd}, J=16.8$ and $4.5 \mathrm{~Hz}, 1 \mathrm{H}), 3.32$ (dd, $J=16.8$ and $14.7 \mathrm{~Hz}, 1 \mathrm{H}), 3.59$ (dd, $J$ $=14.7$ and $4.5 \mathrm{~Hz}, 1 \mathrm{H}), 3.68(\mathrm{~s}, 1 \mathrm{H}), 3.77(\mathrm{~s}, 3 \mathrm{H}), 6.08(\mathrm{~s}$, 1H), 7.07 (d, $J=8.1 \mathrm{~Hz}, 2 \mathrm{H}), 7.13(\mathrm{~d}, J=8.1 \mathrm{~Hz}, 2 \mathrm{H}) ;{ }^{13} \mathrm{C}$ $\mathrm{NMR}\left(\mathrm{CDCl}_{3}, 75 \mathrm{MHz}\right) \delta 18.86,20.99,39.89,49.80,53.49$, $78.91,128.07,129.15,129.25,133.84,137.67,158.63$, 172.29, 197.93; ESIMS $m / z 275\left(\mathrm{M}^{+}+\mathrm{H}\right)$. Anal. Calcd for $\mathrm{C}_{16} \mathrm{H}_{18} \mathrm{O}_{4}$ : C, 70.06; H, 6.61. Found: C, 70.17; H, 6.93.

Compound 2c: 24\%; pale yellow oil; IR (film) 3398, $1699,1610,1499,1293 \mathrm{~cm}^{-1} ;{ }^{1} \mathrm{H} \mathrm{NMR}\left(\mathrm{CDCl}_{3}, 300 \mathrm{MHz}\right) \delta$ $2.22(\mathrm{~s}, 3 \mathrm{H}), 3.59(\mathrm{~s}, 3 \mathrm{H}), 3.79(\mathrm{~s}, 3 \mathrm{H}), 5.76(\mathrm{br} \mathrm{s}, 1 \mathrm{H}), 6.59(\mathrm{~s}$, 1H), 6.81 (br s, 1H), 6.83 (d, $J=8.7 \mathrm{~Hz}, 2 \mathrm{H}), 7.13$ (d, $J=8.7$ $\mathrm{Hz}, 2 \mathrm{H}) ;{ }^{13} \mathrm{C} \mathrm{NMR}\left(\mathrm{CDCl}_{3}, 75 \mathrm{MHz}\right) \delta 12.74,52.09,55.22$, $113.65,113.93,122.56,125.93,129.12,132.48,133.20,141.47$, 144.18, 158.60, 171.27; ESIMS $m / z 289\left(\mathrm{M}^{+}+\mathrm{H}\right)$. Anal. Calcd for $\mathrm{C}_{16} \mathrm{H}_{16} \mathrm{O}_{5}$ : C, 66.66; H, 5.59. Found: $\mathrm{C}, 66.43 ; \mathrm{H}, 5.72$.

Compound 3c: 45\%; white solid, mp 113-114 ${ }^{\circ} \mathrm{C}$; IR (KBr) 3490, 1735, 1672, 1612, 1513, 1438, $1257 \mathrm{~cm}^{-1} ;{ }^{1} \mathrm{H}$ $\mathrm{NMR}\left(\mathrm{CDCl}_{3}, 300 \mathrm{MHz}\right) \delta 1.93(\mathrm{~s}, 3 \mathrm{H}), 2.61(\mathrm{dd}, J=16.8$ and $4.5 \mathrm{~Hz}, 1 \mathrm{H}), 3.30(\mathrm{dd}, J=16.8$ and $14.7 \mathrm{~Hz}, 1 \mathrm{H}), 3.57$ $(\mathrm{dd}, J=14.7$ and $4.5 \mathrm{~Hz}, 1 \mathrm{H}), 3.72(\mathrm{~s}, 1 \mathrm{H}), 3.77(\mathrm{~s}, 3 \mathrm{H}), 3.78$ $(\mathrm{s}, 3 \mathrm{H}), 6.08(\mathrm{~s}, 1 \mathrm{H}), 6.85(\mathrm{~d}, J=8.7 \mathrm{~Hz}, 2 \mathrm{H}), 7.12$ (d, $J=8.7$ $\mathrm{Hz}, 2 \mathrm{H}) ;{ }^{13} \mathrm{C} \mathrm{NMR}\left(\mathrm{CDCl}_{3}, 75 \mathrm{MHz}\right) \delta 18.83,39.98,49.33$, 53.45, 55.08, 78.95, 113.75, 128.84, 129.21 (2C), 158.61, 159.15, 172.23, 197.89; ESIMS $m / z 291\left(\mathrm{M}^{+}+\mathrm{H}\right)$. Anal. Calcd for $\mathrm{C}_{16} \mathrm{H}_{18} \mathrm{O}_{5}$ : C, 66.19; H, 6.25. Found: C, 66.45; H, 6.51.

Compound 2d: $23 \%$; pale yellow solid, mp $156-158{ }^{\circ} \mathrm{C}$; IR (KBr) 3384, 1718, 1603, 1486, $1304 \mathrm{~cm}^{-1}$; ${ }^{1} \mathrm{H}$ NMR $\left(\mathrm{CDCl}_{3}+\mathrm{DMSO}-d_{6}, 300 \mathrm{MHz}\right) \delta 2.25(\mathrm{~s}, 3 \mathrm{H}), 3.59(\mathrm{~s}, 3 \mathrm{H})$, 5.46 (br s, 1H), 5.95 (br s, 1H), $6.63(\mathrm{~s}, 1 \mathrm{H}), 7.17$ (d, $J=8.4$ $\mathrm{Hz}, 2 \mathrm{H}), 7.30$ (d, $J=8.4 \mathrm{~Hz}, 2 \mathrm{H}) ;{ }^{13} \mathrm{C} \mathrm{NMR}\left(\mathrm{CDCl}_{3}+\mathrm{DMSO}-\right.$ $\left.d_{6}, 75 \mathrm{MHz}\right) \delta 12.70,51.51,113.81,122.84,125.22,127.99$, 129.21, 130.72, 132.42, 139.61, 142.38, 144.88, 169.97; ESIMS $m / z 293\left(\mathrm{M}^{+}+\mathrm{H}\right), 295\left(\mathrm{M}^{+}+2+\mathrm{H}\right)$. Anal. Calcd for $\mathrm{C}_{15} \mathrm{H}_{13} \mathrm{ClO}_{4}$ : C, 61.55; H, 4.48. Found: C, 61.49; H, 4.41.

Compound 3d: 48\%; white solid, mp $98-99{ }^{\circ} \mathrm{C}$; IR (KBr) 3497, 1735, 1670, 1492, $1246 \mathrm{~cm}^{-1} ;{ }^{1} \mathrm{H}$ NMR $\left(\mathrm{CDCl}_{3}, 300\right.$ $\mathrm{MHz}) \delta 1.93(\mathrm{~s}, 3 \mathrm{H}), 2.63(\mathrm{dd}, J=16.8$ and $4.8 \mathrm{~Hz}, 1 \mathrm{H})$, $3.29(\mathrm{dd}, J=16.8$ and $14.4 \mathrm{~Hz}, 1 \mathrm{H}), 3.62(\mathrm{dd}, J=14.4$ and $4.8 \mathrm{~Hz}, 1 \mathrm{H}), 3.70(\mathrm{~s}, 1 \mathrm{H}), 3.78(\mathrm{~s}, 3 \mathrm{H}), 6.09(\mathrm{~s}, 1 \mathrm{H}), 7.14(\mathrm{~d}$, $J=8.4 \mathrm{~Hz}, 2 \mathrm{H}), 7.30(\mathrm{~d}, J=8.4 \mathrm{~Hz}, 2 \mathrm{H}) ;{ }^{13} \mathrm{C} \mathrm{NMR}\left(\mathrm{CDCl}_{3}\right.$, 
$75 \mathrm{MHz}) \delta 18.84,39.67,49.35,53.74,78.73,128.65,129.29$, 129.64, 133.93, 135.46, 158.51, 172.19, 197.29; ESIMS $m / z$ $295\left(\mathrm{M}^{+}+\mathrm{H}\right), 297\left(\mathrm{M}^{+}+2+\mathrm{H}\right)$. Anal. Calcd for $\mathrm{C}_{15} \mathrm{H}_{15} \mathrm{ClO}_{4}$ : C, 61.13; H, 5.13. Found: C, 61.47; H, 5.44.

Compound 2e: 24\%; pale yellow oil; IR (film) 3386, $1690,1608,1488,1300 \mathrm{~cm}^{-1} ;{ }^{1} \mathrm{H}$ NMR $\left(\mathrm{CDCl}_{3}, 300 \mathrm{MHz}\right) \delta$ 0.94 (t, $J=7.2 \mathrm{~Hz}, 3 \mathrm{H}), 2.24$ (s, 3H), 4.03 (q, $J=7.2 \mathrm{~Hz}$, 2H), 5.74 (br s, 1H), 6.58 (s, 1H), 6.69 (br s, 1H), 7.18-7.32 $(\mathrm{m}, 5 \mathrm{H}) ;{ }^{13} \mathrm{C} \mathrm{NMR}\left(\mathrm{CDCl}_{3}, 75 \mathrm{MHz}\right) \delta 12.70,13.50,61.46$, $114.02,122.70,125.94,126.94,128.09,128.16,133.11$, 140.73, 141.70, 144.18, 171.01; ESIMS m/z $273\left(\mathrm{M}^{+}+\mathrm{H}\right)$.

Compound 3e: 52\%; pale yellow oil; IR (film) 3495 , $1731,1672,1453,1234 \mathrm{~cm}^{-1} ;{ }^{1} \mathrm{H}$ NMR $\left(\mathrm{CDCl}_{3}, 300 \mathrm{MHz}\right) \delta$ $1.27(\mathrm{t}, J=7.2 \mathrm{~Hz}, 3 \mathrm{H}), 1.94(\mathrm{~s}, 3 \mathrm{H}), 2.65(\mathrm{dd}, J=16.5$ and $4.2 \mathrm{~Hz}, 1 \mathrm{H}), 3.37$ (dd, $J=16.5$ and $14.7 \mathrm{~Hz}, 1 \mathrm{H}), 3.64$ (dd, $J$ $=14.7$ and $4.2 \mathrm{~Hz}, 1 \mathrm{H}), 3.73(\mathrm{~s}, 1 \mathrm{H}), 4.21(\mathrm{q}, J=7.2 \mathrm{~Hz}$, $2 \mathrm{H}), 6.10(\mathrm{~s}, 1 \mathrm{H}), 7.21-7.35(\mathrm{~m}, 5 \mathrm{H}) ;{ }^{13} \mathrm{C} \mathrm{NMR}\left(\mathrm{CDCl}_{3}, 75\right.$ MHz) $\delta 14.00,18.78,39.78,49.93,63.20,78.63,127.92$, 128.34, 128.36, 129.11, 136.88, 158.93, 171.63, 197.93; ESIMS $m / z 275\left(\mathrm{M}^{+}+\mathrm{H}\right)$.

Compound 3f: 59\%; pale yellow oil; IR (film) 3500, $1732,1670,1437,1229 \mathrm{~cm}^{-1} ;{ }^{1} \mathrm{H}$ NMR $\left(\mathrm{CDCl}_{3}, 300 \mathrm{MHz}\right) \delta$ $0.88(\mathrm{t}, J=6.9 \mathrm{~Hz}, 3 \mathrm{H}), 1.17-1.42(\mathrm{~m}, 7 \mathrm{H}), 1.68-1.79(\mathrm{~m}$, $1 \mathrm{H}), 1.90(\mathrm{~s}, 3 \mathrm{H}), 2.23-2.34(\mathrm{~m}, 1 \mathrm{H}), 2.46(\mathrm{dd}, J=16.8$ and $13.5 \mathrm{~Hz}, 1 \mathrm{H}), 2.63(\mathrm{dd}, J=16.8$ and $5.1 \mathrm{~Hz}, 1 \mathrm{H}), 3.84(\mathrm{~s}$, $3 \mathrm{H}), 4.02(\mathrm{~s}, 1 \mathrm{H}), 6.00(\mathrm{~s}, 1 \mathrm{H}) ;{ }^{13} \mathrm{C} \mathrm{NMR}\left(\mathrm{CDCl}_{3}, 75 \mathrm{MHz}\right)$ $\delta 13.90,18.66,22.39,26.37,29.62,31.54,39.46,43.83$, 53.63, 78.05, 129.28, 159.49, 173.52, 197.97; ESIMS $\mathrm{m} / \mathrm{z}$ $255\left(\mathrm{M}^{+}+\mathrm{H}\right)$. Anal. Calcd for $\mathrm{C}_{14} \mathrm{H}_{22} \mathrm{O}_{4}$ : C, 66.12; H, 8.72. Found: C, 66.44; H, 8.56.

Compound 3g: 61\%; white solid, $\mathrm{mp} 89-90{ }^{\circ} \mathrm{C}$; IR (KBr) 3389, 1739, 1657, 1434, $1237 \mathrm{~cm}^{-1}$; ${ }^{1} \mathrm{H} \mathrm{NMR}\left(\mathrm{CDCl}_{3}, 300\right.$ $\mathrm{MHz}) \delta 1.00(\mathrm{~d}, J=6.0 \mathrm{~Hz}, 3 \mathrm{H}), 1.91(\mathrm{~s}, 3 \mathrm{H}), 2.42-2.65(\mathrm{~m}$, $3 \mathrm{H}), 3.86(\mathrm{~s}, 3 \mathrm{H}), 4.06(\mathrm{~s}, 1 \mathrm{H}), 6.00(\mathrm{~s}, 1 \mathrm{H}) ;{ }^{13} \mathrm{C} \mathrm{NMR}\left(\mathrm{CDCl}_{3}\right.$, $75 \mathrm{MHz}) \delta 15.42,18.65,39.03,42.14,53.63,78.45,129.39$, 159.31, 173.29, 197.87; ESIMS $m / z 199\left(\mathrm{M}^{+}+\mathrm{H}\right)$. Anal. Calcd for $\mathrm{C}_{10} \mathrm{H}_{14} \mathrm{O}_{4}$ : C, 60.59; H, 7.12. Found: C, 60.42; H, 7.34.

Compound 2h: 47\%; pale yellow solid, mp 160-161 ${ }^{\circ} \mathrm{C}$; IR (KBr) 3119, 1710, 1611, 1585, 1435, $1298 \mathrm{~cm}^{-1} ;{ }^{1} \mathrm{H}$ NMR $\left(\mathrm{CDCl}_{3}+\mathrm{DMSO}-d_{6}, 300 \mathrm{MHz}\right) \mathrm{d} 2.50(\mathrm{~s}, 3 \mathrm{H}), 3.83(\mathrm{~s}, 3 \mathrm{H})$, 6.70 (br s, 1H), 6.73 (d, $J=8.4 \mathrm{~Hz}, 1 \mathrm{H}), 7.41$ (d, $J=8.4 \mathrm{~Hz}$, $1 \mathrm{H}), 8.90$ (br s, $1 \mathrm{H}) ;{ }^{13} \mathrm{C} \mathrm{NMR}\left(\mathrm{CDCl}_{3}+\mathrm{DMSO}-d_{6}, 75 \mathrm{MHz}\right)$ d 12.94, 51.38, 111.58, 121.82, 123.23, 127.23, 142.98, 147.49, 168.08; ESIMS m/z $183\left(\mathrm{M}^{+}+\mathrm{H}\right)$. Anal. Calcd for $\mathrm{C}_{9} \mathrm{H}_{10} \mathrm{O}_{4}$ : C, 59.34; H, 5.53. Found: C, 59.56; H, 5.78.

Synthesis of Compound 5a. To a stirred solution of $\mathbf{3 a}$ (104 mg, $0.4 \mathrm{mmol})$ in DMSO (1 mL) was added NaI (180 $\mathrm{mg}, 1.2 \mathrm{mmol})$, and the reaction mixture was heated to 120 ${ }^{\circ} \mathrm{C}$ for $30 \mathrm{~min}$. After aqueous extractive workup and column chromatographic purification process (hexanes/EtOAc, 4:1) compounds 5 a was isolated, $45 \mathrm{mg}(56 \%)$.

Compound 5a: yellow oil; IR (film) 3444, 1711, 1602, $1468,1430 \mathrm{~cm}^{-1} ;{ }^{1} \mathrm{H} \mathrm{NMR}\left(\mathrm{CDCl}_{3}, 300 \mathrm{MHz}\right) \delta 2.25(\mathrm{~s}$, $3 \mathrm{H}), 4.75$ (br s, 1H), 4.91 (br s, 1H), 6.56 (d, $J=2.7 \mathrm{~Hz}, 1 \mathrm{H})$, $6.64(\mathrm{~d}, J=2.7 \mathrm{~Hz}, 1 \mathrm{H}), 7.38-7.50(\mathrm{~m}, 5 \mathrm{H}) ;{ }^{13} \mathrm{C} \mathrm{NMR}$ $\left(\mathrm{CDCl}_{3}, 75 \mathrm{MHz}\right) \delta 16.37,113.94,117.28,125.94,127.90$, $128.23,128.99$, 129.27, 137.15, 144.51, 148.56; ESIMS $m / z$
$201\left(\mathrm{M}^{+}+\mathrm{H}\right)$. Anal. Calcd for $\mathrm{C}_{13} \mathrm{H}_{12} \mathrm{O}_{2}$ : C, 77.98; H, 6.04. Found: C, 77.71; H, 6.37.

Acknowledgments. This research was supported by Basic Science Research Program through the National Research Foundation of Korea (NRF) funded by the Ministry of Education, Science and Technology (2010-0015675). Spectroscopic data were obtained from the Korea Basic Science Institute, Gwangju branch.

\section{References and Notes}

1. For the synthesis of Hagemann's ester and related compounds, see: (a) Pollini, G. P.; Benetti, S.; De Risi, C.; Zanirato, V. Tetrahedron 2010, 66, 2775-2802 and further references cited therein. (b) Kingsbury, C. A.; Egan, R. S.; Perun, T. J. J. Org. Chem. 1970, 35, 2913-2918. (c) Gassama, A.; d'Angelo, J.; Cave, C.; Mahuteau, J.; Riche, C. Eur. J. Org. Chem. 2000, 3165-3169. (d) Nour, M.; Tan, K.; Jankowski, R.; Cave, C. Tetrahedron: Asymmetry 2001, 12, 765-769. (e) Nitta, H.; Takimoto, K.; Ueda, I. Chem. Pharm. Bull. 1992, 40, 858-863. (f) Yang, Y.-Q.; Chai, Z.; Wang, H.-F.; Chen, X.-K.; Cui, H.-F.; Zheng, C.-W.; Xiao, H.; Li, P.; Zhao, G. Chem. Eur. J. 2009, 13, 13295-13298.

2. For the synthetic applications of Hagemann's ester, see: (a) Srikrishna, A.; Ramachary, D. B. Tetrahedron Lett. 2000, 41, 2231-2233. (b) Banerjee, M.; Mukhopadhyay, R.; Achari, B.; Banerjee, A. K. Synthesis 2006, 1263-1272. (c) Majetich, G; Allen, S. ARKIVOC 2010, iv, 104-124. (d) Ramachary, D. B.; Ramakumar, K.; Narayana, V. V. J. Org. Chem. 2007, 72, 1458-1463. (e) Ramachary, D. B.; Ramakumar, K.; Narayana, V. V. Chem. Eur. J. 2008, 14, 91439147. (f) Ramachary, D. B.; Narayana, V. V.; Ramakumar, K. Eur. J. Org. Chem. 2008, 3907-3911. (g) Ramachary, D. B.; Narayana, V. V.; Prasad, M. S.; Ramakumar, K. Org. Biomol. Chem. 2009, 7, 3372-3378. (h) Niwas, S.; Bhaduri, A. P. Synthesis 1983, 110-111. (i) Kotnis, A. S. Tetrahedron Lett. 1990, 31, 481-484. (j) Trost, B. M.; Fortunak, J. M. J. Am. Chem. Soc. 1980, 102, 2841-2843.

3. For the oxidation of Hagemann's ester, see: (a) Irie, H.; Katakawa, J.; Tomita, M.; Mizuno, Y. Chem. Lett. 1981, 637-640. (b) Sriyani Ananda, G. D.; Cremins, P. J.; Stoodley, R. J. J. Chem. Soc., Chem. Commun. 1987, 882-883.

4. For our recent results on aerobic oxidation, see: (a) Kim, K. H.; Lee, H. S.; Kim, S. H.; Lee, K. Y.; Lee, J.-E.; Kim, J. N. Bull. Korean Chem. Soc. 2009, 30, 1012-1020. (b) Kim, J. M.; Lee, S.; Kim, S. H.; Lee, H. S.; Kim, J. N. Bull. Korean Chem. Soc. 2008, 29, 2215-2220. (c) Lee, H. S.; Kim, J. M.; Kim, J. N. Tetrahedron Lett. 2007, 48, 4119-4122. (d) Kim, S. C.; Lee, K. Y.; Lee, H. S.; Kim, J. N. Tetrahedron 2008, 64, 103-109. (e) Park, D. Y.; Lee, K. Y.; Gowrisankar, S.; Kim, J. N. Bull. Korean Chem. Soc. 2008, 29, 701-704. For recent examples of aerobic oxidation by other groups, see: (f) Huang, Y.; Gan, H.; Li, S.; Xu, J.; Wu, X.; Yao, H. Tetrahedron Lett. 2010, 51, 1751-1753.

5. When we repeated the reaction of Stoodley (activated charcoal, EtOAc, reflux, $24 \mathrm{~h}$ ), C-1-oxidation product was formed as the major one, as reported. ${ }^{3 b}$

6. Trials for obtaining a crystal for X-ray diffraction failed. ${ }^{3 a}$

7. For the synthesis and synthetic applications of 1-substituted Hagemann's ester derivatives, see: (a) Cui, H.-F.; Yang, Y.-Q.; Chai, Z.; Li, P.; Zheng, C.-W.; Zhu, S.-Z.; Zhao, G. J. Org. Chem. 2010, 75, 117-122. (b) Jung, M. E.; Novack, A. R. Tetrahedron Lett. 2005, 46, 8237-8240. (c) Jung, M. E.; Nishimura, N.; Novack, A. R. J. Am. Chem. Soc. 2005, 127, 11206-11207. (d) Shapland, P. D. P.; Thomas, E. J. Tetrahedron 2009, 65, 4201-4211.

8. For $\mathrm{NaI} / \mathrm{O}_{2}$-assisted concomitant dealkoxycarbonylation/aerobic oxidation, see: (a) Lee, H. S.; Kim, S. H.; Kim, Y. M.; Kim, J. N. Tetrahedron Lett. 2010, 51, 5071-5075. (b) Im, Y. J.; Lee, K. Y.; Kim, T. H.; Kim, J. N. Tetrahedron Lett. 2002, 43, 4675-4678. (c) Santi, R.; Bergamini, F.; Citterio, A.; Sebastiano, R.; Nicolini, M. J. Org. Chem. 1992, 57, 4250-4255. 